\title{
Agravos sensíveis à atenção primária e os aspectos socio-sanitários de criança menores de dois anos
}

\section{Aggrevals sensitive to primary care and socio-sanitary aspects of children under two years}

\author{
Anne Rafaela de Sousa Ribeiro' $\bullet$ Brena Shellem Bessa de Oliveira ${ }^{2} \bullet$ Lara Leite de Oliveira $^{3}$ \\ Emanuella Silva Joventino Melo ${ }^{4}$
}

\begin{abstract}
RESUMO
Objetivo:Verificar associação entre os aspectos sócio-sanitários e os processos de adoecimentos por diarreia e pneumonia em crianças menores de dois anos residentes de um município do Sertão Central do Ceará. Método: Estudo transversal realizado com 94 mães de crianças menores de dois anos nos meses de agosto a outubro de 20I6. Realizaram-se entrevistas estruturadas e análise inferencial por meio dos testes de Qui-quadrado e Linear by linear. Resultados: A diarreia mostrou-se associada com: água utilizada para beber $(p=0,00 \mathrm{I})$, água utilizada para preparação dos alimentos $(p=0,020)$ e contato com o chão/terra $(p=0,0$ II). Já a pneumonia associou-se com: tipo de moradia $(p=0,007)$, forma como é feita a limpeza da casa $(p=0,003)$, água utilizada para beber $(p=0,027)$, tipo de esgoto $(p<0,00 I)$, tipo de descarte do lixo $(p=0,027)$, contato da criança no chão/terra ( $p=0,030)$, limpeza do chão antes das brincadeiras $(p<0,00 \mathrm{I})$ e lavagem das frutas e verduras $(p=0,006)$. Conclusão: Constatou-se que as condições sanitárias podem influenciar o desenvolvimento de condições sensíveis à atenção primária.Assim, os profissionais devem intervir nestas condições para melhor promoção da saúde da criança.
\end{abstract}

Descritores: Saúde da Criança;Atenção Primária a Saúde; Condições sociais.

\begin{abstract}
Objective:To verify the association between the socio-sanitary aspects and the processes of illness due to conditions sensitive to primary care (diarrhea and pneumonia) in children under two years of age living in a municipality in the Central Sertão do Ceará. Method: Cross-sectional study with 94 mothers of children under two years of age from August to October 2016. Structured interviews and inferential analysis were performed using the Pearson's Chi-square and Linear by linear tests. Results: Diarrhea was associated with: water used for drinking $(p=0.00 \mathrm{I})$, water used to prepare food $(p=0.020)$ and contact with soil / soil $(p=0.01 \mathrm{I})$. Pneumonia was associated with: type of housing $(p=0.007)$, how the house was cleaned $(p=$ $0.003)$, water used for drinking $(p=0.027)$, type of sewage $(p<0.00 \mathrm{I})$, type of garbage collection $(p=0.027)$, soil contact $(p$ $=0.030)$, soil cleaning before play $(p<0.00 \mathrm{I})$ and washing of fruits and vegetables $(p=0.006)$. Conclusion: It was found that health conditions may influence the development of conditions sensitive to primary care. Thus, professionals should intervene in these conditions to better promote the child's health.
\end{abstract}

Descriptors: Child Health; Primary Health Care; Social Conditions.

I Enfermeira. Especialista em Pediatria e Neonatologia. Especialista em Saúde da Família. Enfermeira neonatologista do Hospital Regional do Sertão Central. Quixadá. Ceará. Brasil. ORCID: 0000-000I-9625-5232

2 Enfermeira. Doutoranda em Enfermagem. Especialista em Saúde da Família. Bolsista da Coordenação de Aperfeiçoamento de Pessoal de Nível Superior (CAPES). Universidade Federal do Ceará. Fortaleza. Ceará. Brasil. ORCID:0000-000I-6I42-I42I

3 Enfermeira. Doutora em Enfermagem. Docente do Centro Universitário Católica de Quixadá. Quixadá. Ceará. Brasil. ORCID: 0000-000I-6I02-6499

4 Enfermeira. Doutora em Enfermagem. Docente da Universidade da Integração Internacional da Lusofonia Afro-Brasileira. Redenção. Ceará. Brasil. ORCID: 0000000I-9786-5059 


\section{INTRODUÇÃO}

Atualmente, a atenção primária à saúde (APS) é a principal estratégia para a organização do cuidado, constituindo-se como a base estrutural do Sistema Único de Saúde (SUS) no Brasil e como porta de entrada para todos os usuários do serviço sendo capaz de resolver $85 \%$ das demandas advindas da população(l).

Porém, embora se reconheça sua importância, é notória a progressiva desvalorização da APS ao longo dos anos, o que tem a impedido de exercer com eficiência sua função de ordenadora das ações de saúde e coordenadora do cuidado. Esse cenário tem impactado diretamente na assistência integral ao cliente, uma vez que dificulta e fragiliza o processo de prevenção de doenças e promoção de saúde ${ }^{(2)}$.

Assim, surgiu a necessidade de avaliações no âmbito da gestão e na atuação prática da APS, a fim de buscar qualificar a assistência prestada ao usuário nesse nível de atenção. Dentre os indicadores utilizados para essa avaliação, destacam-se as internações por condições sensíveis à atenção primária (ICSAP), que consistem em morbidades que quando atendidas oportunamente na APS podem resolvidas sem a necessidade de hospitalizações ${ }^{(3)}$.

As ICSAP são um reflexo da resolutividade da APS, já que podem ser evitadas com um diagnóstico precoce, com o tratamento adequado de morbidades agudas, acompanhamento e controle de patologias crônicas, as quais são atividades que podem e devem ser realizadas nesse nível de atenção(3).

Diante desse contexto, no ano de 2008, o Ministério da Saúde elaborou a Lista Brasileira de Condições Sensíveis à Atenção Primária que é composta por 19 grupos de diagnósticos, de acordo com a Classificação Internacional de Doenças - CID I0. Essa lista visa analisar os impactos das internações nas condições de saúde e doença dos indivíduos em território nacional, sendo também utilizada como instrumento capaz de investigar o desempenho do sistema de saúde nos três âmbitos federativos $^{(4)}$.

Embora existam esses mecanismos de avaliação da efetividade da atenção prestada ao usuário, ainda é considerável o número de hospitalizações e óbitos infantis em crianças de zero a dois anos por causas evitáveis, as quais são passíveis de serem prevenidas na APS(5).

Além disso, estudo realizado com crianças nos primeiros anos de vida mostrou que no ranking de hospitalizações por CSAP as doenças do aparelho respiratório foram as principais causas, seguidas pelas doenças infecciosas e parasitárias com ênfase na diarreia, gastroenterites virais e outras diarreias não especificadas ${ }^{(6)}$.

As morbidades supracitadas podem ser ocasionadas por uma série de fatores, dentre eles podem-se citar os aspectos social e sanitário nos quais as crianças estão inseridas. Salienta-se que o ambiente é outro determinante importante no processo saúde-doença, sobretudo devido à questão do saneamento básico, qualidade dos alimentos, hábitos e comportamentos de um indivíduo e comunidade $^{(7)}$.

Com base em tais exposições e considerando a carência de estudos relacionados à temática em questão na região do Sertão Central do Ceará, o objetivo deste estudo foi verificar associação entre os aspectos sóciosanitários e os processos de adoecimentos por diarreia e pneumonia em crianças menores de dois anos residentes de um município do Sertão Central do Ceará.

\section{MÉTODO}

Tratou-se de um estudo descritivo, exploratório, transversal com abordagem quantitativa realizado em Quixadá, município que faz parte do semiárido nordestino e está localizado no Sertão Central do Ceará.

Essa pesquisa foi desenvolvida durante as consultas de puericultura em todas as Unidades de Atenção Primária à Saúde (UAPS) da zona urbana $(n=7)$, as quais foram selecionadas por serem responsáveis pelo atendimento do maior número de crianças do município estudado.

A população do estudo foi formada por crianças menores de dois anos de idade residentes na zona urbana de Quixadá e acompanhadas pelo programa de puericultura nas UAPS participantes da pesquisa.

De acordo com o Sistema de Informação da Atenção Básica (SIAB) do referido município, em dezembro de 2015 haviam 1072 crianças de zero a dois anos cadastradas e acompanhadas pelas equipes de saúde da família na zona urbana. Dessas, 546 crianças eram menores de um ano, enquanto que 526 tinham idade que variava de um a dois anos.

Com base nisso foi realizado um cálculo amostral para populações finitas, sendo aplicado um nível de confiança de $90 \%$, uma probabilidade de ocorrer o evento de $50 \%$ e um erro amostral de $10 \%$, totalizando uma amostra de 94 crianças.

Adotou-se como critérios de inclusão: mães e/ou responsáveis de crianças na faixa etária de zero a dois anos de idade acompanhados pelo programa de puericultura das UAPS da zona urbana de Quixadá. Já como critérios de exclusão, adotaram-se: mães e/ou responsáveis que com restrições que os impossibilitassem de responder o formulário de coleta.

Os dados foram coletados nas próprias UAPS nos dias das consultas de puericultura durante os meses de agosto a outubro de 2016, por meio de entrevistas individuais e por conveniência, com a mãe ou responsável pela criança.

Para a obtenção dos dados foi aplicado um formulário estruturado, constituído por questões socioeconômicas, 
sanitárias, aleitamento materno, alimentação complementar e antecedentes de adoecimento da criança. Ressalta-se que para determinar se a criança era acompanhada pelo serviço de saúde, utilizou-se a recomendação do Ministério da Saúde do Brasil ${ }^{(8)}$.

Os dados foram tabulados no programa Microsoft Ex-

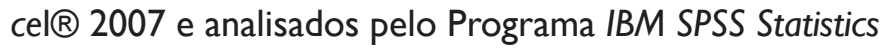
(Versão 20.0), por meio de estatística inferencial utilizando-se os testes Qui-quadrado de Person e linear by linear.

Este estudo foi aprovado pelo Comitê de Ética em Pesquisa do Centro Universitário Católica de Quixadá, conforme parecer de $\mathrm{n}^{\circ} 1.829 .612$ de 22 de novembro de 2016.

\section{RESULTADOS}

Verificou-se que a maioria das crianças participantes deste estudo era do sexo feminino $(n=48 ; 51,1 \%)$; tinha idade entre seis a 12 meses $(n=54 ; 57,4 \%)$, com média de 13,5 meses (DP $\pm 6,16$ ); tinha como principal cuidador a mãe $(n=93 ; 98,9 \%)$; foi amamentado exclusivamente em média 3,8 meses ( $D P \pm 1,99$ ) e estava com o calendário vacinal atualizado $(n=94 ; 100,0 \%)$.

O estudo analisou as possíveis associações entre os aspectos sócio-sanitários nos quais as crianças estão inseridas com algumas condições sensíveis a atenção primária como a diarreia, a pneumonia e outras internações ocorridas de modo geral, como asma, bronquite, viroses, otite e alergias.

A Tabela I destaca a diarreia frente aos aspectos sócio-sanitários. A partir dela, percebe-se que há associação significante entre a diarreia e a água que as crianças utilizam para beber $(p=0,00 \mathrm{I})$, a água utilizada para preparação dos alimentos $(p=0,020)$ e contato com o chão/ terra $(p=0,0 I I)$.

TABELA 1 - Associação entre a diarreia e os aspectos sociais e sanitários em crianças menores de 2 anos.

\begin{tabular}{|c|c|c|c|c|c|}
\hline \multirow[t]{3}{*}{ Variáveis } & \multicolumn{4}{|c|}{ Diarreia } & \multirow[t]{3}{*}{ p-Valor } \\
\hline & \multicolumn{2}{|c|}{ SIM } & \multicolumn{2}{|c|}{ NÃO } & \\
\hline & $\mathrm{N}$ & $\%$ & $\mathrm{~N}$ & $\%$ & \\
\hline Tipo de Moradia & & & & & $0,663^{\mathrm{a}}$ \\
\hline Taipa & 9 & 81,8 & 2 & 18,2 & \\
\hline Tijolo & 63 & 75,9 & 20 & 24,1 & \\
\hline Renda Familiar (em salário mínimo - SM) & & & & & $0,930^{\mathrm{a}}$ \\
\hline Até $2 \mathrm{SM}$ & 65 & 76,5 & 20 & 23,5 & \\
\hline $3-10 \mathrm{SM}$ & 7 & 77,8 & 2 & 22,2 & \\
\hline Cria animal em casa & & & & & $0,269^{\mathrm{a}}$ \\
\hline Sim & 29 & 82,9 & 6 & 17,1 & \\
\hline Não & 43 & 72,9 & 16 & 27,1 & \\
\hline Limpeza da casa & & & & & $0,458^{\mathrm{b}}$ \\
\hline Varre & 4 & 80,0 & 1 & 20,0 & \\
\hline Passa pano com água & 65 & 77,4 & 19 & 22,6 & \\
\hline Lava com água e sabão & 3 & 60,0 & 2 & 40,0 & \\
\hline Água utilizada para beber & & & & & $0,001^{a *}$ \\
\hline Encanada & 1 & 100,0 & 0 & 0,0 & \\
\hline Poço/Cacimba & 26 & 89,7 & 3 & 10,3 & \\
\hline Água de cisterna & 19 & 90,5 & 2 & 9,5 & \\
\hline Água filtrada & 1 & 100,0 & 0 & 0,0 & \\
\hline Água mineral & 25 & 59,5 & 17 & 40,5 & \\
\hline Água para preparação de alimentos & & & & & $0,020^{b} *$ \\
\hline Encanada & 3 & 50,0 & 3 & 50,0 & \\
\hline Poço/Cacimba & 26 & 89,7 & 3 & 10,3 & \\
\hline Água de cisterna & 19 & 90,5 & 2 & 9,5 & \\
\hline Água filtrada & 1 & 50,0 & 1 & 50,0 & \\
\hline Água mineral & 23 & 63,9 & 13 & 36,1 & \\
\hline Tipo de esgoto & & & & & $0,739^{\mathrm{a}}$ \\
\hline Saneamento básico & 41 & 78,8 & 11 & 21,2 & \\
\hline Escorre na rua & 14 & 77,8 & 4 & 22,2 & \\
\hline Fossa & 17 & 70,8 & 7 & 29,2 & \\
\hline Descarte do lixo & & & & & $0,799^{b}$ \\
\hline Carro do lixo & 47 & 78,3 & 13 & 21,7 & \\
\hline Queimado & 24 & 72,7 & 9 & 27,3 & \\
\hline Enterrado & 1 & 100,0 & - & - & \\
\hline Contato com chão/terra & & & & & $0,011^{a *}$ \\
\hline Sim & 63 & 81,8 & 14 & 18,2 & \\
\hline Não & 9 & 52,9 & 8 & 47,1 & \\
\hline Limpeza do chão antes das brincadeiras & & & & & $0,939^{b}$ \\
\hline Sim & 69 & 76,7 & 21 & 23,3 & \\
\hline Não & 3 & 75,0 & 1 & 25,0 & \\
\hline Lavagem de frutas e legumes & & & & & $0,580^{\mathrm{b}}$ \\
\hline Sim & 71 & 76,3 & 22 & 23,7 & \\
\hline Não & 1 & 100,0 & - & - & \\
\hline Lavagem das mãos antes das refeições & & & & & $0,333^{b}$ \\
\hline Sim & 69 & 75,8 & 22 & 24,2 & \\
\hline Não & 3 & 100,0 & - & - & \\
\hline Acompanhamento no serviço de saúde & & & & & $0,102^{\mathrm{a}}$ \\
\hline Sim & 8 & 100,0 & - & - & \\
\hline Não & 64 & 74,4 & 22 & 25,6 & \\
\hline
\end{tabular}

Nota: a teste Qui-quadrado; $b$ teste Linear by linear. ${ }^{*} p<0,05 .{ }^{* *} p<0,0001$. SM na época era $R \$ 880,00$. 
Com base na Tabela 2, a qual mostra o cruzamento entre pneumonia e aspectos sócio-sanitários aos quais as crianças estão inseridas, nota-se que existe relação estatisticamente significante entre essa patologia e o tipo de moradia $(p=0,007)$, a forma como é feita a limpeza da casa $(p=0,003)$, a água utilizada para beber $(p=0,027)$, o tipo de esgoto $(p<0,00 \mathrm{I})$, tipo de descarte do lixo $(p=0,027)$, contato da criança no chão/terra $(p=0,030)$, limpeza do chão antes das brincadeiras $(p<0,00 \mathrm{I})$ e com a lavagem das frutas e verduras $(p=0,006)$.

TABELA 2 - Associação entre a pneumonia e os aspectos sanitários em crianças menores de 2 anos.

\begin{tabular}{|c|c|c|c|c|c|}
\hline \multirow[t]{2}{*}{ Variáveis } & \multicolumn{4}{|c|}{ Pneumonia } & \multirow[t]{2}{*}{ p-Valor } \\
\hline & SIM & & NÃO & & \\
\hline & $\mathrm{N}$ & $\%$ & $\mathrm{~N}$ & $\%$ & \\
\hline Tipo de Moradia & & & & & $0,007^{a *}$ \\
\hline Taipa & 4 & 36,4 & 7 & 63,6 & \\
\hline Tijolo & 7 & 8,4 & 76 & 91,6 & \\
\hline Renda Familiar (em salário mínimo - SM) & & & & & $0,251^{\mathrm{a}}$ \\
\hline Até 2 SM & 11 & 12,9 & 74 & 87,1 & \\
\hline 3-10 SM & - & - & 9 & 100,0 & \\
\hline Cria animal em casa & & & & & $0,206^{a}$ \\
\hline Sim & 6 & 17,1 & 29 & 82,9 & \\
\hline Não & 5 & 8,5 & 54 & 91,5 & \\
\hline Limpeza da casa & & & & & $0,003^{b} *$ \\
\hline Varre & 3 & 60,0 & 2 & 40,0 & \\
\hline Passa pano com água & 8 & 9,5 & 76 & 90,5 & \\
\hline Lava com água e sabão & - & - & 5 & 100,0 & \\
\hline Água utilizada para beber & & & & & $0,027^{\mathrm{b} *}$ \\
\hline Encanada & - & - & 1 & 100,0 & \\
\hline Poço/Cacimba & 7 & 24,1 & 22 & 75,9 & \\
\hline Água de cisterna & 9 & 9,5 & 19 & 90,5 & \\
\hline Água filtrada & - & - & 1 & 100,0 & \\
\hline Água mineral & 2 & 4,8 & 40 & 95,2 & \\
\hline Água para preparação de alimentos & & & & & $0,121^{\mathrm{b}}$ \\
\hline Encanada & - & - & 6 & 100,0 & \\
\hline Poço/Cacimba & 7 & 24,1 & 22 & 75,9 & \\
\hline Água de cisterna & 2 & 9,5 & 19 & 90,5 & \\
\hline Água filtrada & - & - & 2 & 100,0 & \\
\hline Água mineral & 2 & 5,6 & 34 & 94,4 & \\
\hline Tipo de esgoto & & & & & $<0,0001^{a * *}$ \\
\hline Saneamento básico & 2 & 3,8 & 50 & 96,2 & \\
\hline Escorre na rua & 8 & 44,4 & 10 & 55,6 & \\
\hline Armazena em fossa & 1 & 4,2 & 23 & 95,8 & \\
\hline Descarte do lixo & & & & & $0,027^{\mathrm{b} *}$ \\
\hline Carro do lixo & 3 & 5,0 & 57 & 95,0 & \\
\hline Queimado & 8 & 24,2 & 25 & 75,8 & \\
\hline Enterrado & - & - & 1 & 100,0 & \\
\hline Contato com chão/terra & & & & & $0,030^{\mathrm{a} *}$ \\
\hline Sim & 11 & 14,3 & 66 & 85,7 & \\
\hline Não & - & - & 17 & 100,0 & \\
\hline Limpeza do chão antes das brincadeiras & & & & & $<0,0001^{\mathrm{b*}}$ \\
\hline Sim & 7 & 7,8 & 83 & 92,2 & \\
\hline Não & 4 & 100,0 & - & - & \\
\hline Lavagem de frutas e legumes & & & & & $0,006^{\mathrm{b} *}$ \\
\hline Sim & 10 & 10,8 & 83 & 89,2 & \\
\hline Não & 1 & 100,0 & - & - & \\
\hline Lavagem das mãos antes das refeições & & & & & $0,239^{b}$ \\
\hline Sim & 10 & 11,0 & 81 & 89,0 & \\
\hline Não & 1 & 33,3 & 2 & 66,7 & \\
\hline Acompanhamento no serviço de saúde & & & & & $0,284^{\mathrm{b}}$ \\
\hline Sim & - & - & 8 & 100,0 & \\
\hline Não & 11 & 12,8 & 75 & 87,2 & \\
\hline
\end{tabular}

Nota: a teste Qui-quadrado; b teste Linear by linear. ${ }^{*} p<0,05 .{ }^{* *} p<0,0001$. SM na época era $R \$ 880,00$. 
Baseando-se na Tabela 3, percebe-se que a ocorrência de internações prévias vivenciadas pelo público do estudo demonstrou-se associado significantemente com fatores sócio-sanitários, como: tipo de esgoto $(\mathrm{p}=0,0 \mathrm{II})$, contato da criança com o chão $(p=0,027)$ e limpeza do chão antes das brincadeiras $(p=0,031)$.

TABELA 3 - Associação entre as internações e os aspectos sanitários em crianças de 6 meses a 2 anos.

\begin{tabular}{|c|c|c|c|c|c|}
\hline \multirow[t]{2}{*}{ Variáveis } & \multicolumn{4}{|c|}{ Internações } & \multirow[t]{2}{*}{ p-Valor } \\
\hline & SIM & & NÃO & & \\
\hline & $\mathrm{N}$ & $\%$ & $\mathrm{~N}$ & $\%$ & \\
\hline Tipo de Moradia & & & & & $0,160^{\mathrm{a}}$ \\
\hline Taipa & 6 & 54,5 & 5 & 45,5 & \\
\hline Tijolo & 62 & 74,7 & 21 & 25,3 & \\
\hline Renda Familiar (em salário mínimo - SM) & & & & & $0,689^{a}$ \\
\hline Até 2 SM & 62 & 72,9 & 23 & 27,1 & \\
\hline 3-10 SM & 6 & 66,7 & 3 & 33,3 & \\
\hline Cria animal em casa & & & & & $0,113^{\mathrm{a}}$ \\
\hline Não & 46 & 78 & 13 & 22,0 & \\
\hline Sim & 22 & 62,9 & 13 & 37,1 & \\
\hline Limpeza da casa & & & & & $0,160^{\mathrm{b}}$ \\
\hline Varre & 2 & 40,0 & 3 & 60,0 & \\
\hline Passa pano com água & 62 & 73,8 & 22 & 26,2 & \\
\hline Lava com água e sabão & 4 & 80,0 & 1 & 20,0 & \\
\hline Água utilizada para beber & & & & & $0,401^{\mathrm{b}}$ \\
\hline Encanada & 1 & 100,0 & - & - & \\
\hline Poço/Cacimba & 19 & 65,5 & 10 & 34,5 & \\
\hline Água de cisterna & 15 & 71,4 & 6 & 28,6 & \\
\hline Água filtrada & 1 & 100,0 & - & - & \\
\hline Água mineral & 32 & 76,2 & 10 & 23,8 & \\
\hline Água para preparação de alimentos & & & & & $0,855^{\mathrm{b}}$ \\
\hline Encanada & 5 & 83,3 & 1 & 16,7 & \\
\hline Poço/Cacimba & 19 & 65,5 & 10 & 34,5 & \\
\hline Água de cisterna & 16 & 76,2 & 5 & 23,8 & \\
\hline Água filtrada & 2 & 100,0 & - & - & \\
\hline Água mineral & 26 & 72,2 & 10 & 27,8 & \\
\hline Tipo de esgoto & & & & & $0,011^{\text {a* }}$ \\
\hline Saneamento básico & 42 & 80,8 & 10 & 19,2 & \\
\hline Escorre na rua & 8 & 44,4 & 10 & 55,6 & \\
\hline Fossa & 18 & 75,0 & 6 & 25,0 & \\
\hline Descarte do lixo & & & & & $0,203^{b}$ \\
\hline Carro do lixo & 47 & 78,3 & 13 & 21,7 & \\
\hline Queimado & 20 & 60,6 & 13 & 39,4 & \\
\hline Enterrado & 1 & 100,0 & - & - & \\
\hline Contato com chão/terra & & & & & $0,027^{a *}$ \\
\hline Sim & 52 & 67,5 & 25 & 32,5 & \\
\hline Não & 16 & 94,1 & 1 & 5,9 & \\
\hline Limpeza do chão antes das brincadeiras & & & & & $0,031^{\text {b* }}$ \\
\hline Sim & 67 & 74,4 & 23 & 25,6 & \\
\hline Não & 1 & 25,0 & 3 & 75,0 & \\
\hline Lavagem de frutas e legumes & & & & & $0,536^{\mathrm{b}}$ \\
\hline Sim & 67 & 72,0 & 26 & 28,0 & \\
\hline Não & 1 & 100,0 & - & - & \\
\hline Lavagem das mãos antes das refeições & & & & & $0,127^{\mathrm{b}}$ \\
\hline Sim & 67 & 73,6 & 24 & 26,4 & \\
\hline Não & 1 & 33,3 & 2 & 66,7 & \\
\hline Acompanhamento no serviço de saúde & & & & & $0,515^{a}$ \\
\hline Sim & 5 & 62,5 & 3 & 37,5 & \\
\hline Não & 63 & 73,3 & 23 & 26,7 & \\
\hline
\end{tabular}

Nota: a teste Qui-quadrado; b teste Linear by linear. ${ }^{*} p<0,05 .{ }^{* *} p<0,0001$. SM na época era $R \$ 880,00$. 


\section{DISCUSSÃO}

As CSAP podem ser utilizadas para avaliar a resolutividade da APS, porém essas condições, muitas vezes, estão associadas a determinantes socio-sanitários e a comportamentos da população( ${ }^{(9)}$, sendo assim importante investigá-los.

No presente estudo, percebeu-se que o tipo de água consumida pela criança e o tipo de água utilizada para o preparo dos alimentos demonstraram associação significativa com a ocorrência de diarreia infantil. Além disso, notou-se que essa patologia foi mais presente entre as crianças que consumiam água proveniente de poço/cacimba e cisterna. Esse achado corrobora com uma pesquisa que investigou surtos de diarreia na região Nordeste, a qual mostrou que as fontes alternativas (poço, cacimba, cisterna) consistiam nas principais causas de contaminação( ${ }^{(10)}$.

A fonte de água também foi retratada como um importante determinante no desenvolvimento de doenças diarreicas em um estudo desenvolvido no Sul da Ásia, o qual constatou o consumo de água não tratada aumentou os riscos de diarreia infantil( ${ }^{(1)}$.

Sabe-se que baixas condições socioeconômicas afetam diretamente as condições de vida dos indivíduos e dificultam a adoção de hábitos saudáveis ${ }^{(12)}$. Porém, nesta pesquisa não foi encontrada relação significante entre a renda familiar e a ocorrência diarreia, o que se contrapõe ao relatado na literatura ${ }^{(13-14)}$.

Outro fator que demonstrou associação estatisticamente significante com a ocorrência diarreia infantil foi o fato da criança ter contato com o chão ou a terra, sendo que a patologia foi mais recorrente entre as crianças que brincavam na terra em detrimento das que não realizavam essa prática. Esse achado pode estar relacionado com a alta probabilidade do solo ser contaminado com fezes e resíduos que veiculem microorganismos causadores dessa afecção( ${ }^{(15)}$.

Ademais, outra CSAP responsável por uma taxa de morbidade elevada no público infantil é a pneumonia, a qual é apontada como a principal causa de internações em crianças menores de cinco anos em todas as regiões brasileiras $^{(16)}$. A pneumonia apresentou associação estatisticamente significante com o tipo de moradia, sendo menos incidente em crianças que residiam em casa de tijolo. Acredita-se que esse resultado pode ser explicado porque as casas de tijolo tendem a acumular menos poeira e sujidades quando comparadas à casa de taipa e assim, apresentam menor risco de propiciar essa afecção.

$\mathrm{Na}$ amostra estudada não houve associação significante entre pneumonia e renda familiar das crianças, o que diverge de estudo randomizado realizado em São Paulo(17), que constatou que a renda familiar mais elevada consiste em um fator protetor contra a pneumonia.
Ressalta-se que em casas nas quais os cuidadores realizaram a limpeza do chão com pano e água ou com água e sabão antes das crianças brincarem, ocorreu uma menor incidência de pneumonia.Acredita-se que essa relação significante pode estar associada ao fato do uso somente da água ou juntamente com sabão serem mais efetivos na retirada de poeira e resíduos que podem ocasionar patologias no trato respiratório.

Outro determinante que demonstrou-se associado à ocorrência de pneumonia foi o tipo de descarte do lixo da residência, sendo que as crianças que viviam em casas nas quais o descarte do lixo era realizado por carros de lixo ou eram enterrados apresentaram menos episódios de pneumonia quando comparadas àquelas em que o lixo da residência era queimado. Isso ocorre, porque as queimadas e a queima de biomassa geram a poluição do ar atmosférico e, consequentemente, ocasionam danos as vias aéreas e favorecem o desenvolvimento de pneumonia nas crianças ${ }^{(18)}$.

Além disso, a pneumonia também mostrou-se associada estatisticamente com o consumo de água proveniente de fontes alternativas, esgoto à céu aberto, contato da criança com chão/terra e inexistência de lavagem de frutas e verduras ante do consumo.

Averiguar a fonte de água consumida pela criança se faz importante ao se investigar a pneumonia porque existem patógenos causadores dessa afecção, como a Legionella pneumophila, que infectam seres humanos quando esses se expõem à água contaminada ${ }^{(19)}$.

Assim, ressalta-se a importância de se buscar meios que evitem a contaminação da água que é ingerida pela criança e da que é usada para o preparo de alimentos, a fim de evitar a contaminação cruzada. Dentre esses métodos enfatiza-se a realização da lavagem de frutas e verduras com água potável antes da ingestão, a realização de métodos caseiros de tratamento da água, como, por exemplo, a fervura e a realização do armazenamento destes em local higienizado.

É sabido que o saneamento ambiental inadequado é capaz de gerar muitos impactos negativos na saúde das crianças por proporcionar o desenvolvimento de diversas doenças infecto-parasitárias que culminam no aumento das taxas de internações infantis ${ }^{(7)}$, apesar disso, notou-se que as crianças deste estudo que tinham acesso à saneamento básico apresentaram taxas mais elevadas de internações.

Diante desses achados, acredita-se que o número de internações prévias das crianças podem ter propiciado a mudança de hábitos dos cuidadores no que concerne à higiene do ambiente e à exposição das crianças à terra a fim de evitar esses eventos. Essa suposição é levantada, uma vez que as mães não foram indagadas se essas condutas iniciaram-se antes ou após os episódios de interna- 
mento.Além disso, ressalta-se que as patologias que ocasionam hospitalizações infantis são multifatoriais, assim, seria importante a realização de uma análise multivariada para maiores esclarecimentos a cerca desses resultados.

Por fim, destaca-se que embora diversos fatores possam predispor processos de adoecimentos na primeira infância, a atenção primária se configura como um instrumento crucial para agir buscando investigar, intervir e minimizar as ICSAP por meio de uma cobertura efetiva e realização de atividades de educativas voltadas para a prevenção de doenças e promoção da saúde ${ }^{(20)}$.

\section{CONCLUSÃO}

O presente estudo identificou que as crianças da pesquisa, mesmo com toda a cobertura e assistência da atenção primária a saúde, ainda estão susceptíveis a diarreia e a pneumonia.Além disso, essas condições demonstraram-se associadas a algumas variáveis sócios-sanitárias, como: a água ingerida pelas crianças e utilizada na preparação dos alimentos destas crianças, contato com o chão/terra, o tipo de moradia, a forma como é feita a limpeza da casa, o tipo de esgoto, tipo de descarte do lixo, limpeza do chão antes das brincadeiras e lavagem das frutas e verduras.

Diante dos resultados desta pesquisa, acredita-se que ela possa contribuir para a elaboração, implantação e avaliação de novas estratégias desenvolvidas pela APS, as quais visem potencializar as ações de prevenção de doenças e promoção da saúde da criança no seu território adscrito. Além disso, pode ainda ser útil para o aumento conhecimento clínico, epidemiológico e crítico do enfermeiro durante sua prática clínica, uma vez que Ihe fornece subsídios para direcionar e qualificar o cuidado à criança.

Por fim, destaca-se como limitação o fato de o estudo ter sido desenvolvido de forma unicêntrica e com amostragem por conveniência. Assim, ressalta-se a importância de serem realizados estudos longitudinais nesse campo, de forma a investigar por um maior período as ICSAP e direcionar o cuidado prestado a esse público. 


\section{REFERÊNCIAS}

I. Brasil. Ministério da Saúde. Política Nacional de Atenção Básica. Brasília (DF): Ministério da Saúde, 2017. Portaria $N^{\circ}$ 2.436, de 21 de setembro de 2017. Disponível em: http:// pesquisa.in.gov.br/imprensa/jsp/visualiza/index.jsp?jornal $=I$ \&data $=22 / 09 / 201$ \& \&agina $=68$

2. Morosini MVGC, Fonseca AF. Reviewing the Brazilian National Primary Healthcare Policy at such a time?. Cad Saúde Pública [Internet]. 2017 [acesso em 04 jun 2020]; 33(I):e002063 I6. Disponível em: http://dx.doi.org/ I0.1590/ $0102-311 \times 00206316$

3. Santos BV, Lima DS, Fontes CJ. Hospitalization for ambulatory care-sensitive conditions in the state of Rondônia, Brazil: descriptive study for the period 2012-2016. Epidemiol Serv Saude [Internet]. 2019 [acesso em 04 jun 2020]; 28(I):e2017497. Disponível em: http://dx.doi.org/I0.5 I23/ sl679-4974201900010000।

4. Brasil. Ministério da Saúde. Lista Brasileira de Internações por Condições Sensíveis à Atenção Primária. 2008. Disponível em: http://bvsms.saude.gov.br/bvs/saudelegis/sas/2008/ prt022I_17_04_2008.html

5. Santos ILF, Gaíva MAM, Abud SM, Ferreira SMB. Hospitalização de crianças por condições sensíveis à atenção primária. Cogitare Enferm [Internet]. 2015 [acesso em 04 jun 2020]; 20(I):I7|-9. Disponível em: http://dx.doi.org//0.5380/ce. v20il.37586

6. Silva VLS, França GVA, Santos IS, Barros FC, Matijasevich A. Characteristics and factors associated with hospitalization in early childhood: 2004 Pelotas (Brazil) birth cohort. Cad Saúde Pública [Internet]. 2017 [acesso em 04 jun 2020]; 33(10): e000357/6. Disponível em: http://dx.doi.org/I0.1590/0102-31 Ix000357I6

7. Siqueira MS, Rosa RS, Bordin R, Nugem RC. Hospitalizations due to diseases associated with poor sanitation in the public health care network of the metropolitan region of Porto Alegre, Rio Grande do Sul State, Brazil, 20I02014. Epidemiol Serv Saúde [Internet]. 2017 [acesso em 04 jun 2020]; 26(4):795-806. Disponível em: http://dx.doi. org/|0.5 | 23/s | 679-497420 I 70004000 I I

8. Brasil. Ministério da Saúde. Secretaria de Atenção à Saúde. Departamento de Atenção Básica. Saúde da criança : crescimento e desenvolvimento / Ministério da Saúde. Secretaria de Atenção à Saúde. Departamento de Atenção Básica. Brasília : Ministério da Saúde, 2012. Disponível em: https:// bvsms.saude.gov.br/bvs/publicacoes/saude_crianca_crescimento_desenvolvimento.pdf

9. Lima RCDSM, Gama MEA, Lima RDSM. Condições sensíveis à atenção primária em hospital de referência pediátrica no Maranhão. Rev Pesq Saúde [Internet]. 2017 [acesso em 04 jun 2020]; I8(2): 97-I0I. Disponível em: http://www.periodicoseletronicos.ufma.br/index.php/revistahuufma/article/ viewFile/5744/5 198

10. Rufino R, Gracie R, Sena A, Freitas CM, Barcellos C. Diar- rhea outbreaks in northeastern Brazil in 2013, according to media and health information systems - Surveillance of climate risk and health emergencies. Ciênc Saúde Coletiva [Internet]. 2016 [acesso em 04 jun 2020]; 2 I(3):77788. Disponível em: http://dx.doi.org//0./590//4/381232015213.17002015

I I. Hasan MM, Richardson A. How sustainable household environment and knowledge of healthy practices relate to childhood morbidity in South Asia: analysis of survey data from Bangladesh, Nepal and Pakistan. BMJ Open [Internet]. 2017 [acesso em 05 jun 2020]; 7:e0 I50 I9. Disponível em: http:// dx.doi.org/l0.1 I36/bmjopen-2016-015019.

I2. Pazó RG, Frauches DO, Maria, CBM, Cade NV. Modelagem hierárquica de determinantes associados a internações por condições sensíveis à atenção primária no Espírito Santo, Brasil. Cad Saúde Pública [Internet]. 2014 [acesso em 04 jun 2020]; 30(9):|89|-1902. Disponível em: http://dx.doi. org/10.1590/0102-31।X00099913

13.André SCS, Santos APM,Veiga TB, Takayanagui AMM. Influência das desigualdades econômicas e sanitárias na incidência da diarreia infantil em Minas Gerais, 20 I0. Rev baiana saúde pública [Internet]; 2016 [acesso em 04 jun 2020]; 40(3): 785-795. Disponível em: https://doi.org//0.22278/23/82660.2016.v40.n3.a779

14. Oliveira RKL, Oliveira BSB, Bezerra JC, Silva MJN, Melo FMS, Joventino ES. Influence of socio-economic conditions and maternal knowledge in self-effectiveness for prevention of childhood diarrhea. Esc Anna Nery [Internet]. 2017 [acesso em 04 jun 2020]; 2 I (4):e20 I6036I. Disponível em: http://dx.doi.org/ |0.1590/2 I77-9465-ean-20 I6-036 I

I5. Simões BS, Machado-Coelho GLL, Pena JL, Freitas SN. Condições Ambientais e Prevalência de Infecção parasitária em Xukuru-Kariri Indígenas, Caldas, Brasil. Rev Panam Salud Publica [Internet]. 2015 [acesso em 04 jun 2020]; 38( I):42-8. Disponível em: https://www.scielosp.org/article/ rpsp/20 I 5.v38n I/42-48/

16. Pereira FJR, Silva CC, Lima Neto EA. Profile of Hospitalization for Primary Care Sensitive Conditions subsidizing health activities in Brazilian regions. Saúde Debate. [Internet] 2015 [acesso em 04 jun 2020]; 40(107): I008-17. Disponível em: http://www.scielo.br/scielo.php?script=sci_arttext\&pid=SO I 03- I I 0420 I 50004010087

17. Pina JC, Moraes AS, Freitas ICM, Mello DF. Role of Primary Health Care in child hospitalization due to pneumonia: a case-control study. Rev Latino-Am Enfermagem [Internet]. 2017 [acesso em 04 jun 2020]; 25:e2892. Disponível em: http://dx.doi.org//0.1590/I5 |8-8345.1731.2892.

18. Machin AB, Nascimento LFC. Effects of exposure to air pollutants on children's health in Cuiabá, Mato Grosso State, Brazil. Cad Saúde Pública [Internet]. 2018 [acesso em 04 jun 2020]; 34(3):e000066l7. Disponível em: http://dx.doi. org/I0.1590/0102-31 Ix00006617

19. Inostroza E, PINTO R. Neumonía por agentes atípicos en 
niños. Rev Med Clin Condes [Internet]. 2017 [acesso em 04 jun 2020]; 28(I): 90-96. Disponível em: https://doi.org/l0.1016/j.rmclc.2017.01.006

20. Costa JSD, Pattussi MP, Morimoto T, Arruda JS, Bratkowski GR,Sopelsa $M$ et al.Tendência das internações por condição sensivel à atenção primária e fatores associados em Porto
Alegre, RS, Brasil. Ciênc Saúde Coletiva [Internet]. 2016 [acesso em 05 jun 2020]; 21 (4): I289-96. Disponível em: http://dx.doi.org/I0.1590/|4|3-8I2320|52|4.150420I5.

Recebido: 2020-07-05 Aceito: 2020-07-31 